\title{
Investigation of the Dynamics Bed Shear Stress Distribution around a Circular Cylinder Using Various Turbulences Models
}

\author{
Nazila Kardan ${ }^{1, *}$, Habib Hakimzadeh ${ }^{2}$, and Yousef Hassanzadeh ${ }^{3}$ \\ 1 Department of Civil Engineering, Azarbaijan Shahid Madani University, Tabriz, Islamic Republic of Iran \\ 2 Faculty of Civil Engineering, Sahand University of Technology, Tabriz, Islamic Republic of Iran \\ 3 Faculty of Civil Engineering, University of Tabriz, Tabriz, Islamic Republic of Iran \\ *E-mail: n.kardan@azaruniv.ac.ir (Corresponding author)
}

\begin{abstract}
Scouring around cylinders is greatly affected by the value of bed shear stress entered on sediment materials of the bed. Regarding the importance of the bed shear stress fluctuations and their influence on the initiation and development of scouring around cylinders, in this paper, the dynamic bed shear stress distributions around a circular cylinder in high Reynolds numbers flow is investigated using various turbulence closures. Since the bed shear stress can be regarded as one of the key parameters in scouring and erosion predictions, then the accurate calculation of this parameter would be the first step in scour prediction and calculation. As the vortex shedding at the downstream of a cylinder depends on the turbulence intensity of flow, a similar behavior may be anticipated for the dynamic bed shear stress. To evaluate the bed shear stress distribution, several numbers of numerical simulations are conducted to predict the bed shear stress using the CFD solver FLUENT. Various turbulence models of time-and space-averaged types are used to simulate the turbulence effects. Regarding the importance of the turbulence models in accurate simulation of the cylinder wake region, three models of $k-\varepsilon$, RSM and LES are evaluated using a comparison of the flow pattern and the fluctuation of near wake vortices. Results revealed that in the simulation with LES turbulence model, the wake remains very symmetrical, consisting primarily of two large vortices in the near wake at two sides of the cylinder. With time passing, a transition occurs between the two states, from the initially symmetrical to the asymmetrical. Also, comparing the critical bed shear stress pattern in different phases show that the bed shear stress is repeated again after a certain time period. This is in good agreement with the fluctuation time period of wake vortices.
\end{abstract}

Keywords: Cylinder, vortex shedding, bed shear stress, turbulence closures, numerical simulation. 


\section{Introduction}

Scour is a natural phenomenon, caused by the erosive power of the flowing water over alluvial beds in rivers and streams. It occurs when the critical bed shear stress for initiation of sediment particle transport is exceeded locally [1]. Local scour around bridge piers is a complex three dimensional phenomenon, which occurs as a result of the strong flow-sediment-pier interaction. The main cause of the sediment movement in river beds is the bed shear stress exerted by the turbulent incoming flow. Understanding these interactions between the flow and sediment particles is in importance to completely know the scouring mechanism and its reduction [2].

In river beds, the sedimentary materials that affected by the turbulences of flow meet movements which are completely random and may change in time and place. The probable fluxes of forces on bed sediment particles are the main cause of this phenomenon. The random movements of sediment particles reveal that the direction and the amount of sediment particle's movements completely depend on the turbulence intensity and flow characteristics. The Complexity of flow around sediment particles causes a little information can be found in the field of nature and feature of fluxes of forces acted on the particles. In primary studies conducted in this field, the Gaussian function was basically provided to predict the distribution pattern of bed shear stress [3-4]. Paintal (1971) was the first researcher that employed the Gaussian function to predict the non-dimensional bed shear stress distribution and Shields number [5]. Cheng and Chiew (1998) used the Gaussian function to obtain the velocity distribution along the flow depth. It is stated that the amount of lift forces entered on sediment particles is a function of shear stress and velocity distribution [6].

Cheng and Law (2003) showed that predicting the distribution of bed shear stress using a Gaussian function cannot yield accurate results for different types of sediment beds so that a new Lognormal function was applied by them to all fields [7]. It was concluded that this new function causes an increasing in the accuracy of achieved results is considered. Therefore, based on these studies the importance of performing such studies about the dynamic bed shear stress is clearly revealed. However, in a few studies, the effect of force fluctuations on the movement of sediment particles is considered.

Researchers like Einstein (1950) [8] Bagnold (1973) [9] and Yalin (1977) [10] evaluated the movement of sediment particles only under the influence of averaged value of flow parameters. But Grass and Ayoub for the first time in 1983 investigated the influence of bed shear stress fluctuations on the movement of sediment particles, and demonstrated that the value of forces resulted from the fluctuations of bed shear stress and caused the movement of sediment particles, is more than the effect of the averaged value of these forces [11]. In their experimental studies, they studied scouring under two conditions of clear-water and live-bed considering the effects of velocity fluctuations and turbulence as well as bed shear stress. In line with these studies, Sumer et al. (2003) obtained similar results and showed that the movement of sediment particles under the effect of forces entered on the particles, is much more than the averaged amount [12]. They also investigated the effect of turbulence intensity on the fluctuations of bed shear stress as well as the movement of sediment materials.

In another investigation conducted by Nicholas (2001) the 2D turbulent characteristics of open channel flow are investigated using the CFD code FLUENT. The main object of this research is to study the turbulent characteristics on two types of the gravel bed. The effect of boundary roughness is considered by applying the two models; the wall function approach and the random elevation model. Also, the sensitivity of the numerical model analyzed respect to the horizontal and vertical mesh resolution. The results have been presented based on the variation of "Turbulent Viscosity" and "Turbulent Kinetic Energy" respect to the boundary roughness of "y/h" [13]. In a paper presented by Pascale et al. (2004) the bed shear stress around deflectors experimentally estimated using different methods of log profile, drag, Reynolds and turbulent kinetic energy over sand beds. Also, three boundary roughness are considered. The investigated methods include: reach-average bed shear stress $\left(\tau_{o}=\rho g R S_{f}\right)$, quadratic stress law $\left(\tau_{o}=\rho C_{d} U^{2}\right)$, Reynlolds stress $\left(\tau_{0}=-\rho\left\langle u^{\prime} w^{\prime}\right\rangle\right)$, and turbulent kinetic energy $\left(\tau_{0}=C_{1}\left[0.5 \rho\left(\left\langle u^{\prime 2}\right\rangle+\left\langle v^{\prime 2}\right\rangle+\left\langle w^{\prime 2}\right\rangle\right)\right]\right)[14]$.

The value of bed shear stresses entered on sediment particles greatly affected the scouring around cylinders. According to the Shields criteria, the sediment particles around obstructions start moving when the bed shear stress enacted by the turbulence flow become larger than the critical bed shear stress. Also, the variation in the value of bed shear stress occurred as a function of secondary flows. In the flow field around cylinders, two secondary flows occurred; the first secondary flow, which observed in front of the cylinder called horseshoe vortex, and the secondary one known as wake vortices behind the cylinder in which caused 
by the flow separation at the two sides of the cylinder. The existence of such secondary flows causes occurring intensive fluctuations in the value of bed shear stress nearby the cylinders. On the other hand, the flow separation and occurrence of vortex shedding, which fluctuate at near wake region, lead to fluctuating of the bed shear stress at two sides and downstream of the cylinder. As a result, the movement of the sediment particles observed in these regions [15].

As it is obvious from published papers about bed shear stress that there are few researchers that focused on the effect of bed shear stress distribution and its variations on scouring mechanism. Investigating the available researchers in the field of pier scouring showed that the scouring mechanism strongly affected by the flow fluctuations, vortex shedding from the sides of the pier, and the bed shear stress. On the other hand, understanding the role of bed shear stress in the initiation and development of the scour around piers will lead to getting suitable design methods and countermeasures which may significantly reduce or even prevent the bed erosion. Considering the importance of bed shear stress fluctuations and its effect on the initiation and development of scouring around cylinders, it is tried in this paper to analyze the bed shear stress distribution and its value in different phases of fluctuations. To do this, CFD solver FLUENT software is used for numerical simulation of the three-dimensional flow field nearby the circular cylinder. To achieve desirable numerical results, different turbulence closures are investigated to reach the more accurate result for simulation of the flow turbulence effects.

\section{Materials and Methods}

\subsection{Governing Equations}

The governing equations of the turbulent flows are the unsteady, incompressible, filtered time-averaged Navier-Stokes equations [16]:

$$
\begin{gathered}
\frac{\partial \rho}{\partial t}+\frac{\partial}{\partial x_{i}}\left(\rho u_{i}\right)=0 \\
\frac{\partial}{\partial t}\left(\rho u_{i}\right)+\frac{\partial}{\partial x_{j}}\left(\rho u_{i} u_{j}\right)=-\frac{\partial p}{\partial x_{i}}+\frac{\partial \tau_{i j}}{\partial x_{j}}+\rho g_{i}
\end{gathered}
$$

Where $t$ is time, $u_{i}$ is the velocity in $i$ direction, $p$ is the total pressure, $g_{i}$ is the gravity acceleration in $i$ direction, $\rho$ is fluid density and $\tau_{i j}$ shows the stress tensor defined as follows [17]:

$$
\begin{gathered}
\tau_{i j}=\rho v\left(\frac{\partial u_{i}}{\partial x_{j}}+\frac{\partial u_{j}}{\partial x_{i}}\right)-\rho \overline{u_{i}^{\prime} u_{j}^{\prime}} \\
-\rho \overline{u_{i}^{\prime} u_{j}^{\prime}}=\rho v_{t}\left(\frac{\partial u_{i}}{\partial x_{j}}+\frac{\partial u_{j}}{\partial x_{i}}\right)-\frac{2}{3} k \delta_{i j}
\end{gathered}
$$

Here $v$ and $v_{t}$ are molecular and turbulent viscosity respectively, $k$ is turbulence kinetic energy and $\delta_{i j}$ is Kronecker delta [10]. The only difference between the momentum equation in turbulent and laminar flows is the $-\rho \overline{u_{i}^{\prime} u_{j}^{\prime}}$ term, which is referred to eddy shear stress or turbulent stress. This term is not physically a stress; however, it indicates the effects of inertia (momentum) exchanges [18].

\subsection{Turbulence Models}

Turbulent Reynolds stress in time-averaged equations can be closed by applying any of the several turbulence closures. No single turbulence closure is accepted universally for solving all types of turbulent problems, however, selecting a suitable model for a special case depends on the needed accuracy of the results and the nature of the flow field. The $\kappa-\varepsilon$ model is the simplest and most widely used two-equation turbulence closure that solves two separate transport equations and provides an independent determination of the turbulent kinetic energy $\kappa$ and its dissipation rate $\varepsilon$ [19]. The Reynolds stress model (RSM) provides closure of the Reynolds-averaged Navier-Stokes equations for determining the Reynolds stresses by solving transport equations and providing the energy dissipation rate by another equation [20,21].

The RSM calculates the effects of circulation, vorticity, streamline curvature, and rapid changes in the strain rate in a more efficient method than the two-equation eddy viscosity turbulence models. The RSM is preferred to the other eddy viscosity turbulence models in a simulation of the complex flows, however, it 
needs extensive computational effort and time. Large-eddy simulation (LES) is an alternative method in which the averaging RANS or conventional time method is not adopted with additional transport equations being solved to obtain the Reynolds stresses that resulted from the process of averaging. In LES, only the large scale motions called large eddies which occurred in turbulent flows are directly simulated, and the motions with sub-grid scales (SGS) are not directly modeled so that computational cost is significantly reduced in comparison to DNS. It is concluded that LES is more accurate than the RANS methods because the large eddies that contain most of the turbulent energy and are responsible for most of the turbulent mixing and momentum transfer, mostly simulated in this turbulence model. Furthermore, it is confirmed that in turbulence flows, the small scales tend to be more homogeneous and isotropic than the large ones, and thus modeling the sub-grid scale motions is easier than modeling all scales of motions within a single model in the RANS approach. Therefore, currently LES is the most viable/promising numerical approach to simulate the realistic turbulent flows [21, 22].

\subsection{FLUENT CFD Solver}

In the present study, the CFD solver FLUENT is used to solve the three-dimensional Reynolds-averaged Navier-Stokes equations in unsteady incompressible flow. In FLUENT, the governing equations solved sequentially using the control volume method. To construct discretized algebraic equations for the dependent variables, the equations are integrated over each control volume so that the discretized equations are linearized using an implicit method [18].

Appropriate conditions must be considered as the boundaries at computational domain boundaries depending on the flow nature. In simulations performed in this study, two separate inlets and two separate outlets respectively at the upstream and downstream end are specified (Fig. 1). At the inlet boundary (left), the transverse and the vertical velocity (w) components are set to be zero. At the outlet (right), turbulent quantities in the flow direction and the gradients of the velocity are set to zero and a reference pressure of zero is given [23]. At the two sides and the top boundaries, zero normal velocity and zero normal gradients of all variables are applied by defining a symmetrical boundary condition. No-slip boundary condition is applied to the other solid boundaries. At the bed boundary, the standard wall-function boundary condition is implemented $[24,25]$. Use of wall functions reduces the size of the required meshes at near-wall boundaries and results in improved computational efficiency. At the water surface boundary, rigid lid boundary condition is applied.

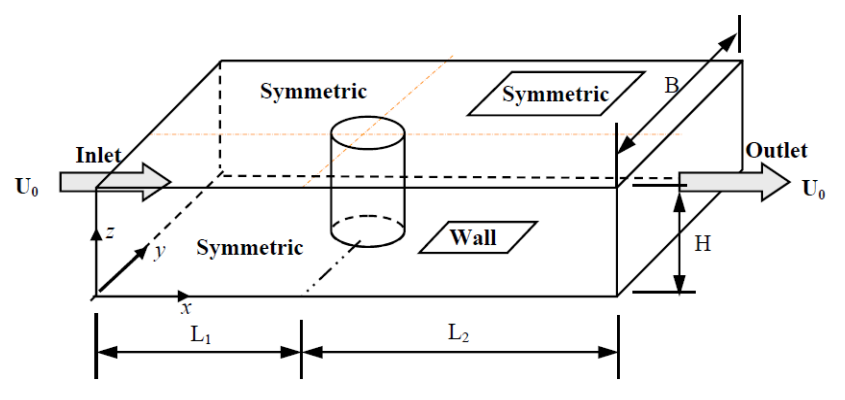

Fig. 1. Defined boundaries at different surfaces of the computational domain.

\section{Numerical Validation}

To validate the three-dimensional numerical results, the characteristics of the model examined in Melville (1975) experiments have been conducted. The flume was $19 \mathrm{~m}$ length, $0.456 \mathrm{~m}$ width and $0.15 \mathrm{~m}$ water depth with a constant discharge of $0.0171 \mathrm{~m}^{3} / \mathrm{s}$ [15]. A weir of $0.085 \mathrm{~m}$ height has been installed at the flume outlet to provide the flow depth of $0.15 \mathrm{~m}$. The depth-averaged velocity was set to be $0.25 \mathrm{~m} / \mathrm{s}$. The circular cylinder with $0.051 \mathrm{~m}$ diameter and a hydraulically smooth surface was placed at the center of flume in sediment bed. The median particle size was $0.385 \mathrm{~mm}$ with a uniformity parameter of (the sediment gradation) of 1.5. The specific grain density of the sediment was 2.66 and angle of repose of the sand was $32^{\circ}$. The flow velocity (V) was determined to be 0.92 of the critical velocity $\left(V_{o r}\right)$ for initial movement of sediment particles. The $V_{o r}$ obtained $27.1 \mathrm{~cm} / \mathrm{s}$, corresponding to $\theta_{c r}=0.030$. The flow in the flume had a Reynolds number of about 90,000 and Froude number of 0.2. 
To perform numerical simulation with CFD solver of FLUENT, the computational domain is firstly meshed. To investigate the effect of cell numbers in the computational domain, the free surface level is evaluated by comparison of three cell numbers of 35000, 50000 and 80000, and the results compared with the experimental data of Melville [15]. In Fig. 2, the variation of water free surface has been presented. The results confirm that up to a certain value of cell numbers, the free surface shows considerable variations, however, after this certain value no significant change is observed in free surface.

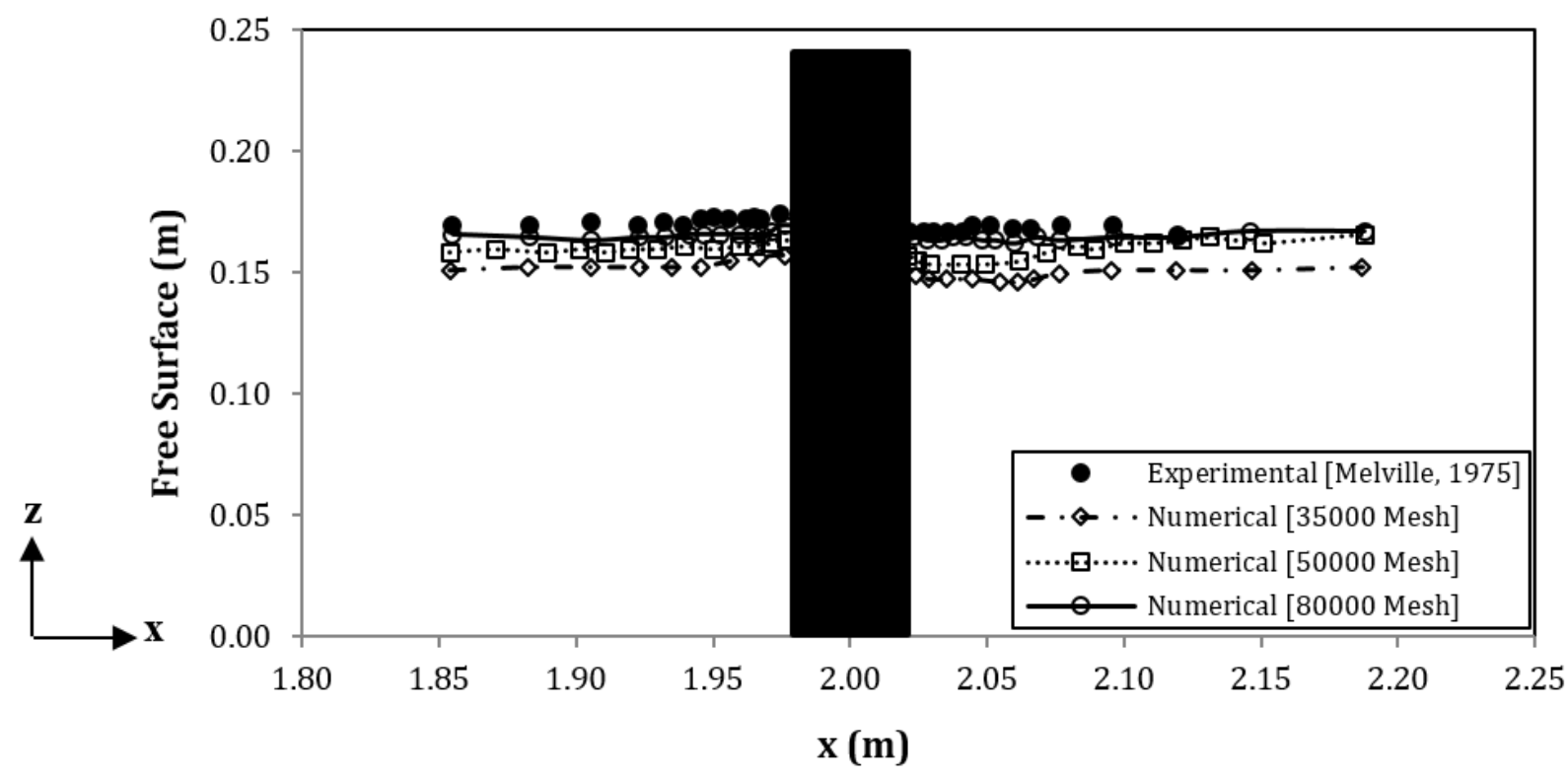

Fig. 2. The effect of cell numbers on variation of water free surface.

Thus, for this value, a good agreement is achieved between the numerical and experimental data. It should be considered that using finer cells and increasing the cell number over this value, only lead to increasing the simulation time. So, approximately 80000 cell numbers are provided, in which the cells in three directions of length, width and height set to be 190x26x16 respectively. According to the cell size, the time step is selected 0.001 seconds. The computed root mean square error (RMSE) for three numbers of cell size is presented in Table 1 to show the effect of cell number on variations of free surface.

Table 1. Comparison of the RMSE value of different mesh numbers.

\begin{tabular}{cccc}
\hline Cell number & 35000 & 50000 & 80000 \\
\hline RMSE & 0.478 & 0.145 & 0.0727 \\
\hline
\end{tabular}

Considering the influence of meshing type on numerical results, two types of meshing called structured and unstructured are evaluated. In turbulent flows with high Reynolds number, the vortex shedding process begins when the separation occurred in the boundary layer of the cylinder. The separated boundary layer rolls up into the vortices and begins to move into wake region. The vortices continue to grow roughly symmetrically when they are fed vorticity from the boundary layer. Then, the wake undergoes a transition and starts to become strongly asymmetrical. In using structured meshing, the wake remains symmetrical and does not experience the transition. In unstructured meshing, however, the transition occurred in the wake and the asymmetrical form of vortices appeared at the wake region. Fig. 3 shows the wake region in structured and unstructured meshing. So, an unstructured type of meshing is provided for other numerical simulations. 


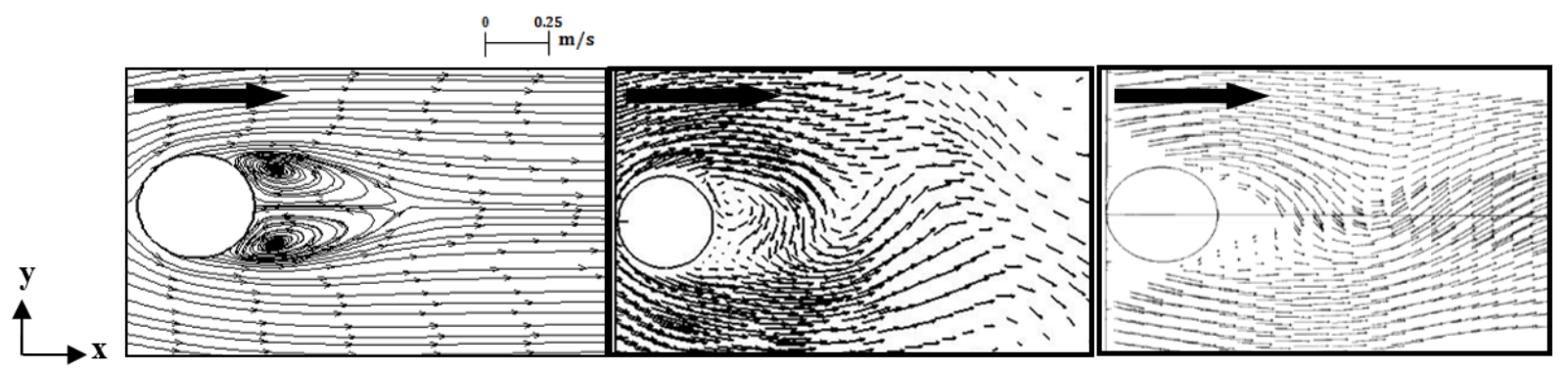

Fig. 3. Comparison of the flow pattern around a circular cylinder (a) structured mesh (b) unstructured mesh (c) Melville experimental data.

\section{Results and Discussion}

\subsection{Comparison of Turbulence Models}

The simulated wake region using standard $k-\varepsilon$, is in a symmetrical form, which as mentioned, is not accurate for the high turbulent flow around the cylinder. In the simulation with RNG model, at the earlier time of simulation, the transition occurred and the wake starts to become strongly asymmetrical. Later, contrary to what was thought, the state of periodic shedding is not simulated and the vortices had no interaction in the wake region. So that, the flow is considered almost as a steady stream in the far wake of the cylinder. Regarding the importance of the vortex dynamics and their effects on the fluctuations of bed shear stress, $k-\varepsilon$ model may not be an appropriate turbulence closure for simulation of such high turbulent flows.

In the simulation with the RSM turbulence model, the asymmetrical form and the interaction of vortices (vortex fluctuations) are completely simulated. However, fluctuation of near wake vortices is not periodic and a certain value cannot be extracted for a period of vortex shedding. In the LES turbulence model, at very early times, the wake remains very symmetrical and consisting of two large vortices in the near wake at two sides of the cylinder. With time passing, a transition occurs between the two states, from the initially symmetrical to the asymmetric one. For the time period of this phenomenon, the different values have been proposed by different researchers. The asymmetrical form of vortices in initial stages of assimilation is presented in Fig. 4. This model has capable of reproducing random fluctuating vortices in addition to any periodic fluctuating vortices. It is concluded that the time period of vortex shedding is about 8 seconds $(\mathrm{T}=8 \mathrm{~s})$ and their frequency is calculated about $0.78 \mathrm{~Hz}$. In Fig. 5, shedding of the vortices in a time period of 8 seconds has been shown in different fluctuating phases. These results are in good agreement with ones achieved in Sumer et al. (2003) [12] study. They show that an accurate simulation of bed shear stress depends on strongly exact applying of the turbulence effects by turbulence closures.

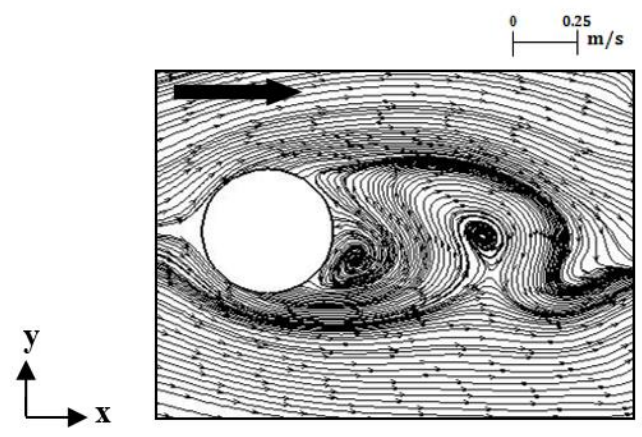

Fig. 4. Asymmetrical pattern of wake vortices in LES turbulent model.

\subsection{Verification of Bed Shear Stress in Different Fluctuating Phases}

Based on the accurate assimilation of vortex fluctuations and shedding in the near wake of the cylinder provided by LES turbulence closure, this model is applied to investigate the fluctuations of bed shear stresses. 
The time period for analyzing the bed shear stress fluctuations is determined about 8 seconds based on the time period of vortices shedding at wake region. Fig. 6, shows the different phases of bed shear stress fluctuations. It should be explained that the figure at the left side shows two areas, firstly, the area that the value of bed shear stress is larger than the critical value (highlighted with red color), and secondly is the area that the bed shear stress has lower value than the critical one (highlighted with white color). In figure displayed in the right side, the picture shows the contours of non-dimensional bed shear stress which may be larger or lower than the critical value of $0.196 \mathrm{~N} / \mathrm{m}^{2}$. The critical bed shear stress is calculated by formula of $\tau=u_{*_{c}}^{2} \times 1000, u_{*_{c}}^{2}=\theta_{c r}(S-1) g d_{50}$.

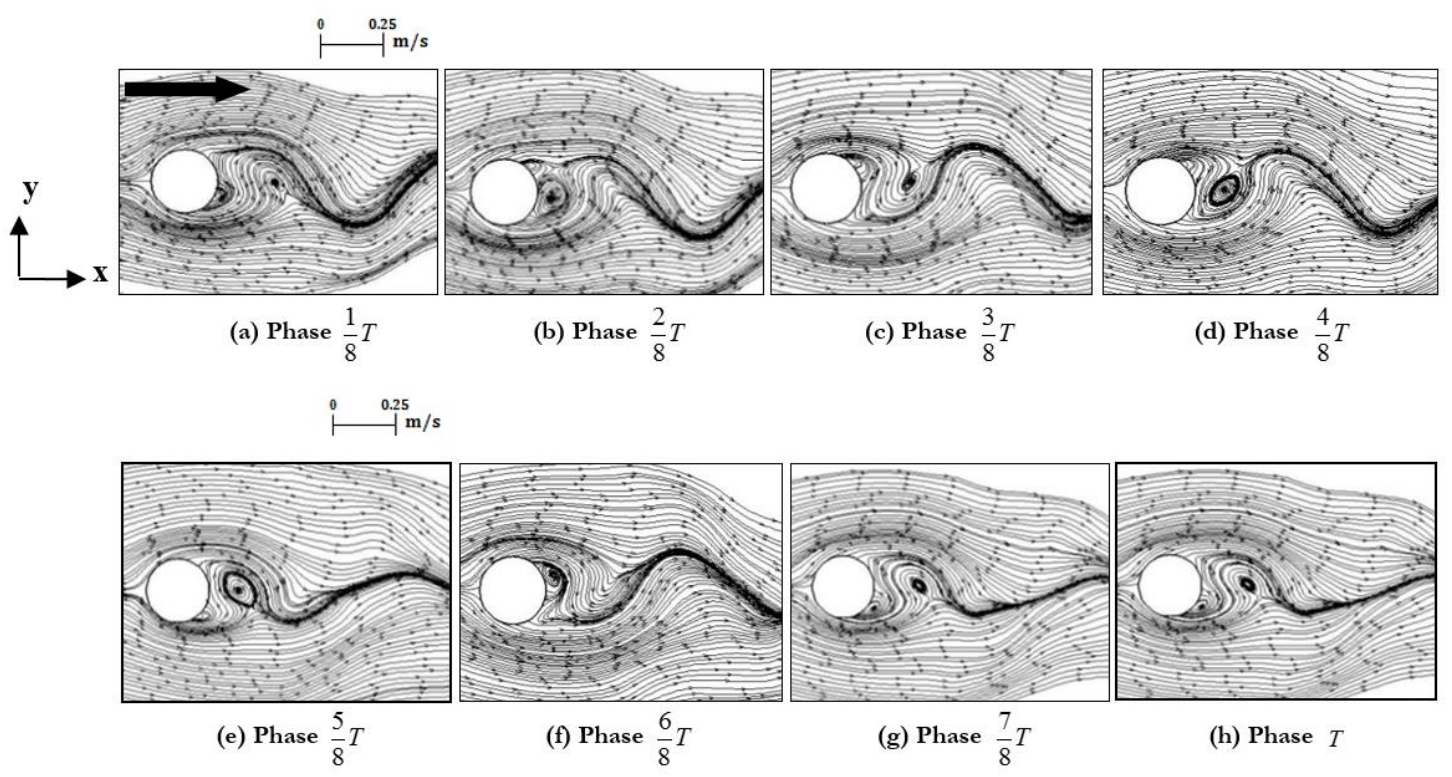

Fig. 5. The vortex shedding process in different fluctuating phases using the LES turbulence model.

These phases are according to the vortex fluctuation phases in Fig. 5. The pattern of vortex shedding is described for the first time by Perry et al. (1982) [1] using particle paths. In the present study, more accurate description of particle paths and shedding phases are presented. In Perry et al. (1982) shedding of vortices is discussed using particle paths, however, in this paper, further investigations are conducted about vortex shedding from one side to another site of the cylinder and near wake region. During the first time of shedding process, at $t=0$, the cavity in near wake is open and the flow paths do not penetrate into the cavity. In phase of $1 / 8 T$, the anticlockwise circulation from the lower side of the wake enters into the cavity. At this time, the maximum critical bed shear stress is measured just below the point that the first circulation is generated. At $t=4 / 8 T$, this circulation is shed into the downstream region and the critical bed shear stress decreased at the lower side of the wake. At $t=4 / 8 T$, the clockwise circulation from the upper side of the wake penetrates into the cavity. At this time, by decreasing the bed shear stress in the lower side, increasing in bed shear stress value occurred at the upper side below the clockwise second. This circulation becomes larger and simultaneously shed, so that, decreasing in bed shear stress observed at the upper side of the cylinder. Comparison of the critical bed shear stress patterns in two phases, namely $1 / 8 T$ and $T+1 / 8 T$ revealed that the bed shear stress repeated again after a certain time period which equals 8 seconds. This is in good agreement with the fluctuation time period of wake vortices shown in Fig. 5. So, based on presented results, it is concluded that the fluctuations of bed shear stress are according to the fluctuations of the vortex shedding. 

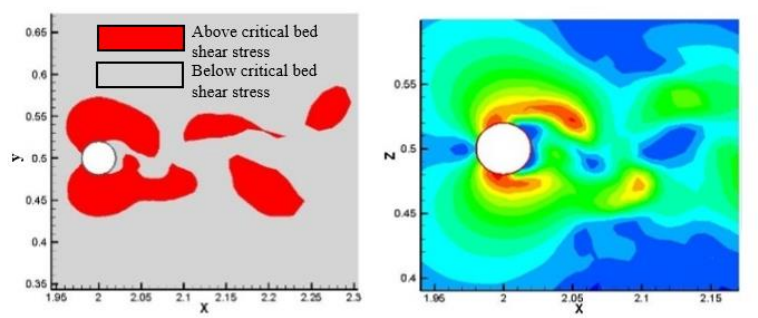

(a) Phase $\frac{1}{8} T$
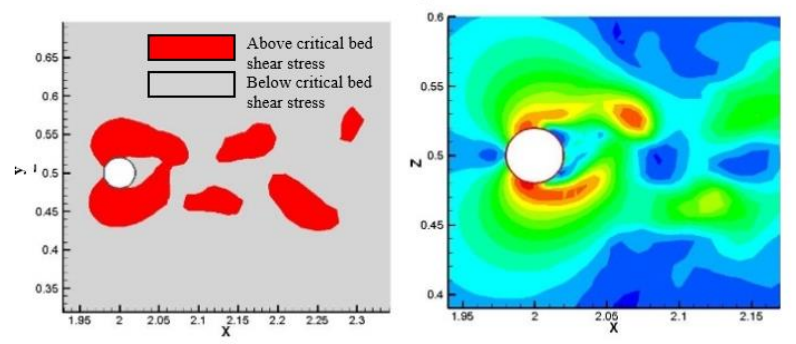

(c) Phase $\frac{3}{8} T$
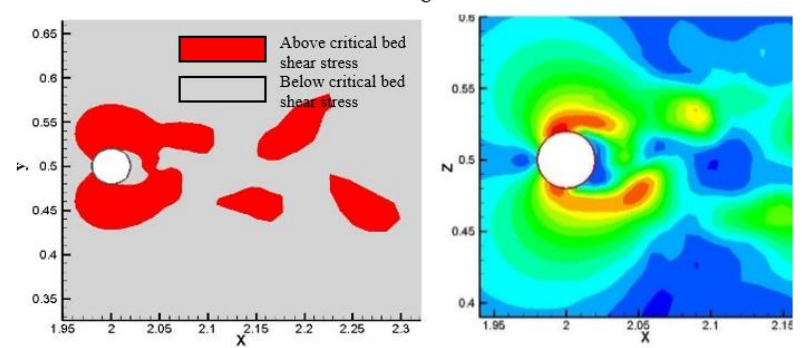

(e) Phase $\frac{5}{8} T$
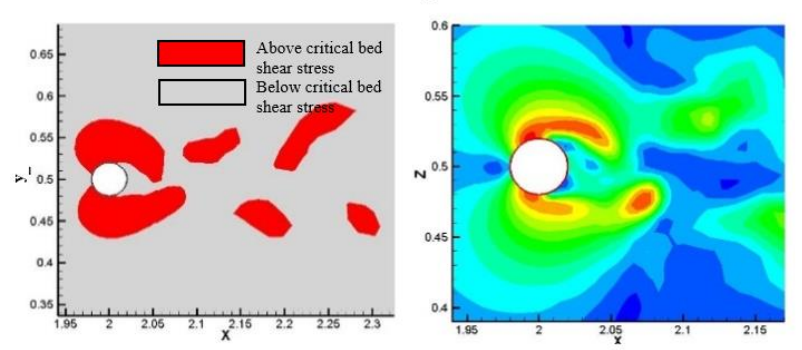

(g) Phase $\frac{7}{8} T$

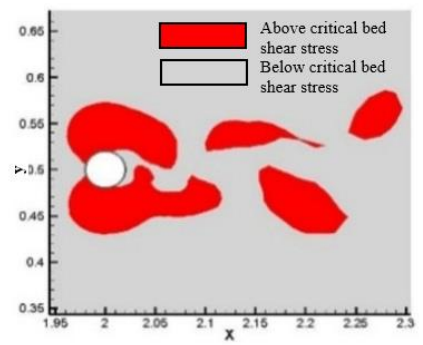

(i) Phase $T+\frac{1}{8} T$
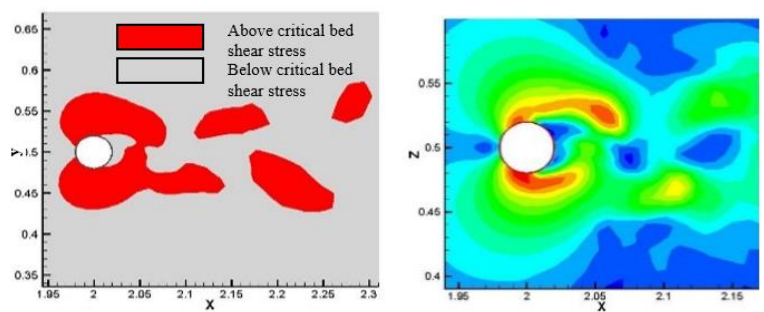

(b) Phase $\frac{1}{4} T$
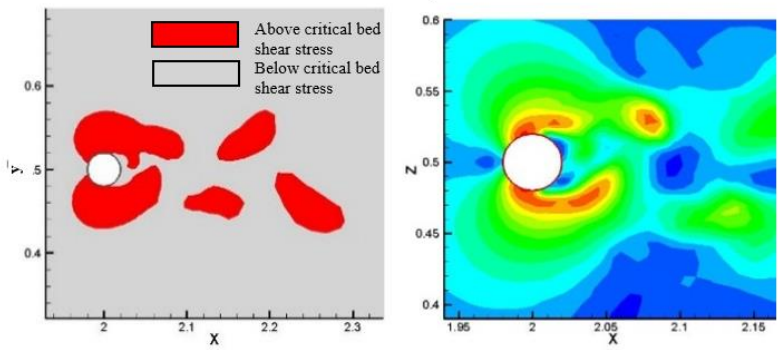

(d) Phase $\frac{1}{2} T$
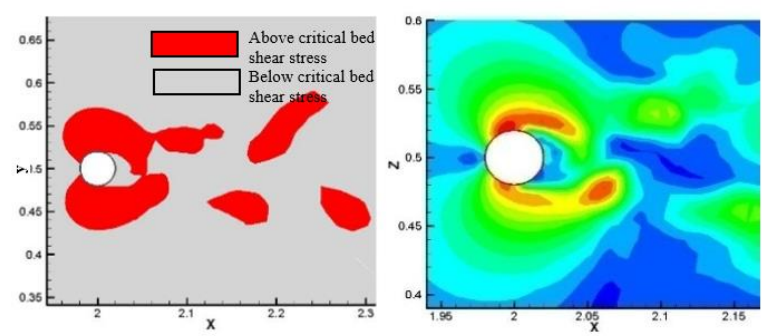

(f) Phase $\frac{3}{4} T$
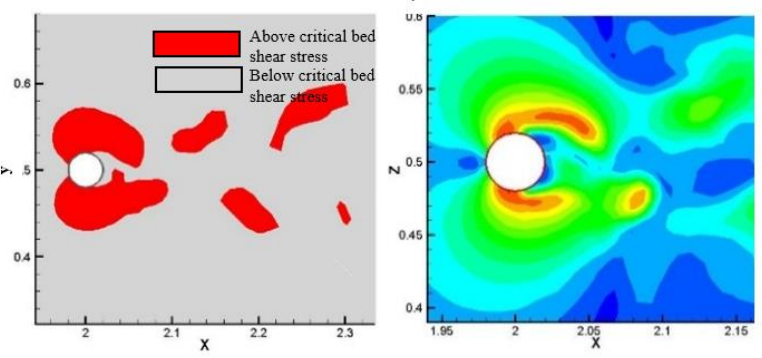

(h) Phase $T$

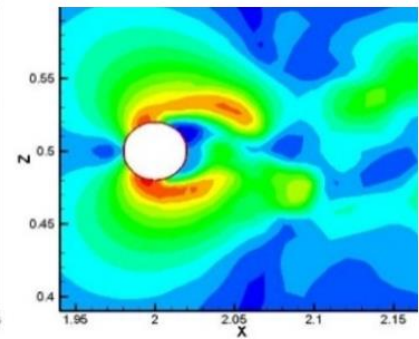

Fig. 6. Bed shear stress in different fluctuations phases in a period time of 8 seconds.

Figure 7 shows the predicted phases of bed shear stresses and that is averaged value during the running time of the simulation. As observed in this figure, in the initial steps of flow development, the bed shear stress varied progressively according to the fluctuations of wake vortices and after a certain time period, which is approximately 70 seconds in the present study, at the wake region established a certain fluctuation 
pattern which is obvious in this figure. In Fig. 8, it is presented the bed shear stress fluctuation for the first 8 seconds (72 80) after flow development and establishment of the equilibrium state. The time averaged value of non-dimensional bed shear stress is about 3.520 respects to the critical one. In second 8 periods (80 88) shown in Fig. 9, the time averaged value of bed shear stress is 3.553 and in the third 8 seconds (88 96) it equals 3.560 .

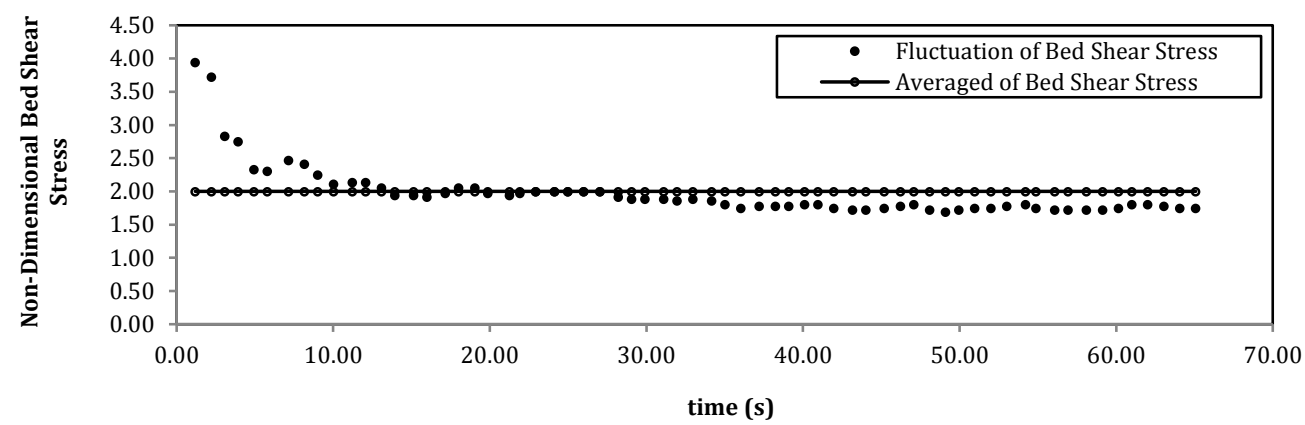

Fig. 7. Fluctuations of the non-dimensional bed shear stress during the numerical simulation.

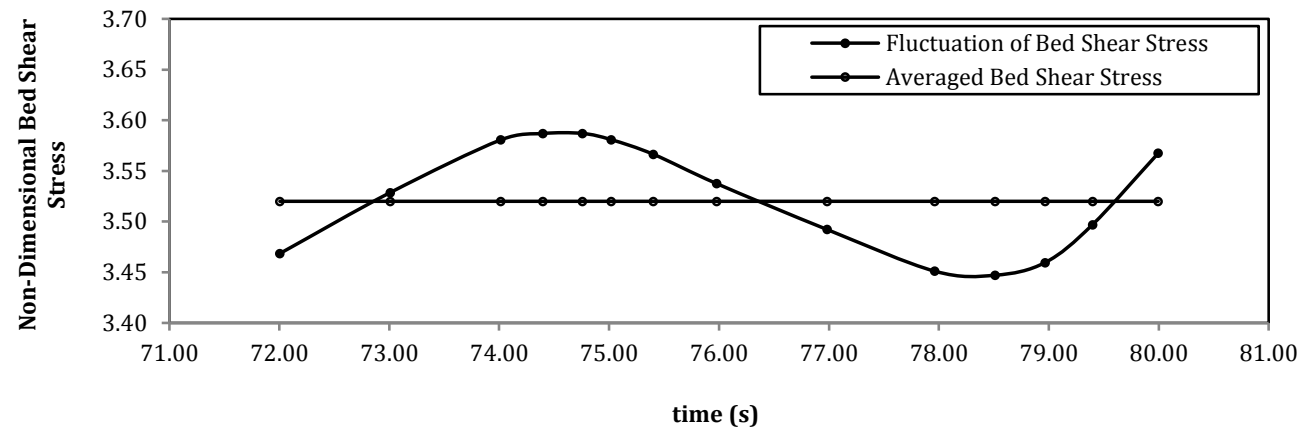

Fig. 8. Fluctuations of the non-dimensional bed shear stress in first 8 seconds.

As shown in Fig. 10, the averaged value is about 3.558 in third 8 seconds. Considering the negligible differences between the averaged values of bed shear stresses in first, second and third time periods, it is concluded that after a complete development of the flow, the predicted pattern of bed shear stress in a certain time period, is a sinusoidal curve. Comparing the results with Cheng and Law (2003) [7] ones confirmed that applying the effects of flow turbulence can yield an accurate and acceptable form of bed shear stress distribution for different types of sediment beds. Using Gaussian function, however, to predict the distribution of shear stress cannot be applied to all fields. The distribution pattern of shear stress also is in good agreement with Cheng and Chiew (1998) [6] distribution pattern which obtained from the velocity distribution profile. So, it is concluded that predicting the distribution of dynamic bed shear stress using a shedding pattern of wake vortex can be the best method among models applied to predict the dynamics of shear stress.

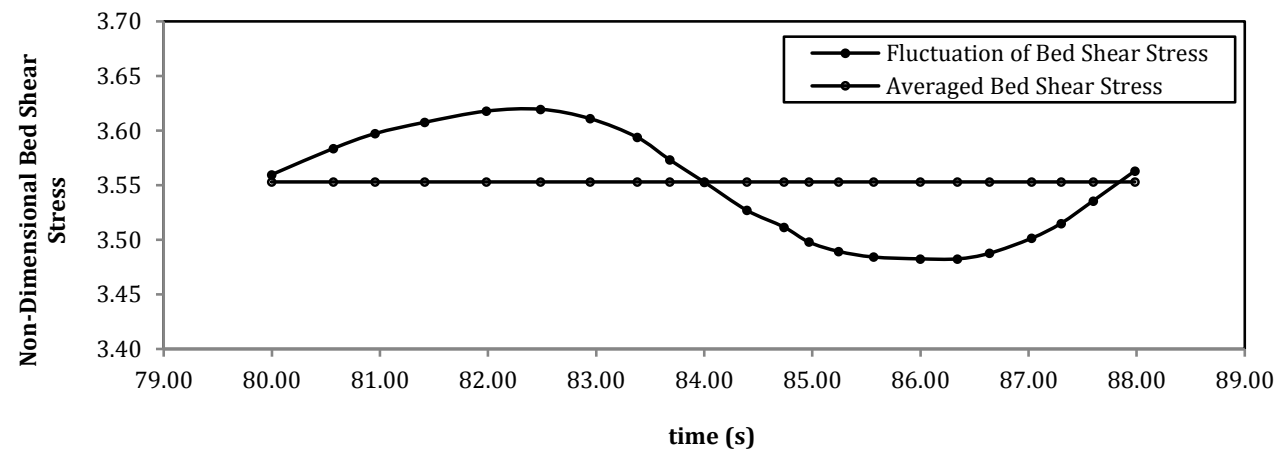

Fig. 9. Fluctuations of the non-dimensional bed shear stress in second 8 seconds. 


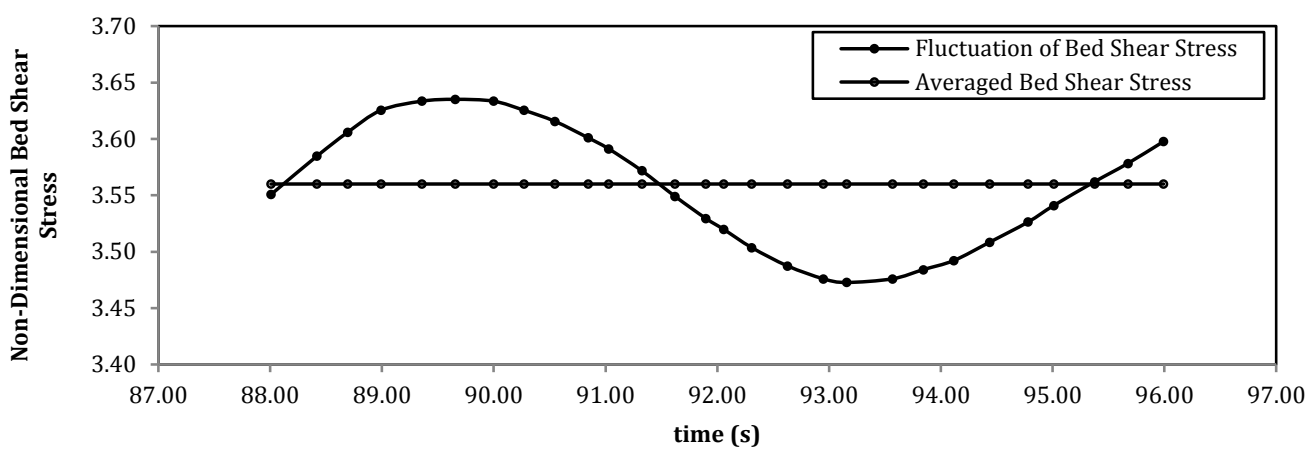

Fig. 10. Fluctuations of the non-dimensional bed shear stress in third 8 seconds.

As a second important result extracted from this research, based on the fluctuations of the vortex shedding and the bed shear stress, the scouring mechanism can be known from another view. Different researchers showed that two parameters are the main cause of scouring mechanism; down-flow at the cylinder upstream and flow separation and wake vortices at the sides and downstream of the cylinder. However, Kardan et al. (2016) showed that the importance of the flow separation is much more than the flow-down, in which it is the main reason that causes the scour initiates from the sides of the pier and develops to the downstream and upstream. On the other hand, the vortex shedding is resulted from the flow separation. Increasing the velocity around the cylinder and the flow separation, result in considerable variation in value and distribution of bed shear stress. At the first time from flow developing (to 55 seconds at Fig. 7), the bed shear stress forced random movements on sediments, but after passing about 55 seconds, the bed shear stress gets a sinusoid form according to the vortices shedding. It can be concluded that if the flow separation and as a result, the vortices shedding at the two sides of the pier, are decreased, the bed shear stress reduced considerably along decreasing the flow separation. Consequently, the exerted forces on the bed sediments is decreased in which this decreasing lead to control the scouring mechanism.

\section{Conclusion}

In the present study, the vortex shedding at the near wake of a circular cylinder is simulated and more evaluated using three turbulence models of $k-\varepsilon$, RSM and LES. Regarding to the importance of the mesh type in achieving accurate results in numerical simulation, two types of structured and unstructured meshing are evaluated by comparisons of the flow pattern. Based on the numerical simulations, the following conclusions are deduced:

Comparison of the flow pattern in structured and unstructured meshing shows that using the unstructured mesh lead to achieve accurate results. This is due the fact that the simulated flow in Melville (1975) study is a turbulent flow with a relatively high Reynolds number, which causes an asymetrical wake region is observed. In applying structured mesh, the simulated wake region is symmetrical, which is not in coinciding with the experimental one.

By using the two turbulence models of $k-\varepsilon$ and RSM, the vortices at the near wake of the cylinder have simulated as symmetrical pattern. In the LES model, however, asymmetrical shedding of vortices simulated in good agreement with the experimental one. So, simulation of the flow turbulences using LES turbulence closure lead to accurate results.

Comparing the fluctuations of the vortices and bed shear stresses demonstrates that the phases of bed shear stress fluctuation are in good agreement with the vortices fluctuation phases at the near wake region.

The time period of the vortices shedding computed about 8 seconds with a frequency of $0.78 \mathrm{~Hz}$. Regarding to the adoption of the bed shear stress and vortex shedding phases, the time period of the bed shear stress will be 8 seconds too.

It is confirmed that the flow separation has a very important role in the initiation and development of the scouring around piers. The flow separation increases the forces exerted on the sediments and causes movements of them. Also, the flow separation is the main reason of the wake vortices behind the pier. So, it is concluded that investigation of the development and shedding of the vortices at the near wake region of the cylinders can yield very useful information about the nature and variation of the bed shear stresses. 
Understanding the mechanism of the flow separation, wake region, the bed shear stress, and the interaction between these three parameters, lead to present design methods and new countermeasures that significantly prevent the scouring around cylinders.

\section{References}

[1] A. E. Perry, M. S. Chong, and T. T. Lim, "The vortex shedding process behind two-dimensional bluff bodies," Journal of Fluid Mechanics, vol. 116, pp. 77-90, 1982.

[2] Z. P. Zang, F. P. Gao, and J. S. Cui, "Physical modelling and swirling strength analysis of vortex shedding from near bed piggyback pipeline," Applied Ocean Research, vol. 40, pp. 50-59, 2013.

[3] Z. H. Yang, "Large-eddy simulation: Past, present and the future," Chinese Journal of Aeronautics, vol. 28, no. 1, pp. 11-24, 2015.

[4] Z. H. Song, M. Duan, and J. Gu, "Hydrodynamics and vortex shedding characteristics of two tandem cylinders of different diameters in a steady flow," Ships and Offshore Structures, vol. 10, no. 5, pp. 1-14, 2015.

[5] A. S. Paintal, "A stochastic model of bed load transport," Journal of Hydraulic Research, vol. 4, pp. 527554, 1971.

[6] N. S. Cheng and Y. M. Chiew, "Pickup probability for sediment entrainment," Journal of Hydraulic Engineering, ASCE, vol. 124, no. 2, pp. 232-235, 1998.

[7] N. S. Cheng and A. W. K. Law, "Fluctuations of turbulent bed shear stress," Journal of Engineering Mechanics, ASCE, vol. 129, no. 1, pp. 126-130, 2003.

[8] H. A. Einstein, "The bed-load function for sediment transportation in open channel flows," U.S. Dept. of Agriculture, Washington, 1951.

[9] R. A. Bagnold, "The nature of saltation and of 'bed-load' transport in water," Proceedings of the Royal Society of London, Series A (Mathematical, Physical and Engineering Sciences), vol. 332, no. 1591, 1973.

[10] M. S. Yalin, Mechanics of Sediment Transport, 2nd ed. Oxford; New York: Pergamon Press, 1977.

[11] A. J. Grass and R. N. M. Ayoub, "Bed load transport of fine sand by laminar and turbulent flow," in Proceedings of the 18th Coastal Engineering Conference, Cape Town, South Africa, B. L. Edge, Ed. American Society of Civil Engineers, New York, 1983, pp. 1589-1599.

[12] B. M. L. Sumer, H. C. Chua, N. S. Cheng, and J. Fredsøe, "Influence of turbulence on bed load sediment transport," Journal of Hydraulic Engineering, ASCE, vol. 129, no. 8, pp. 585-596, 2003.

[13] A. P. Nicholas, "Computational fluid dynamics modelling of boundary roughness in gravel-bed rivers: An investigation of the effects of random variability in bed elevation," Earth Surface Processes and Landforms, vol. 26, no. 4, pp. 345-362, 2001.

[14] M. B. Pascale, R. Colleen, F. L. Michel, and J. G. Susan, "Comparing different methods of bed shear stress estimates in simple and complex flow fields," Earth Surface Processes and Landforms, vol. 29, no. 4, pp. 1403-1415, 2004.

[15] B. W. Melville, "Local scour at bridge sites," Dept. of Civil Engineering, School of Engineering, Univ. of Auckland, Auckland, New Zealand, Rep. No. 117, 1975.

[16] F. M. White, Viscose Fluid Flow, 2nd ed. McGraw-Hill: New York, 1991.

[17] B. E. Launder and D.B. Spalding, "The numerical computation of turbulent flows," Computer Methods in Applied Mechanics and Engineering, vol. 3, no. 2, pp. 269-289, 1974.

[18] T. M. Salaheldin, J. Imran, and H. Chaudhry, "Numerical modeling of three-dimensional flow field around circular piers," Journal of Hydraulic Engineering, ASCE, vol. 130, no. 2, pp. 91-100, 2004.

[19] B. E. Launder, G. J. Reece, and W. Rodi, "Progress in the development of a Reynolds-stress turbulence closure," Journal of Fluid Mechanics, vol. 68, no. 3, pp. 537-0566, 1975.

[20] M. M. Gibson and B. E. Launder, "Ground effects on pressure fluctuations in atmospheric boundary layer," Journal of Fluid Mechanics, vol. 86, no. 3, pp. 491- 511, 1978.

[21] S. J. Lee, J. H. Lee, and J. C. Suh, "Numerical investigation on vortex shedding from a hydrofoil with a beveled trailing edge," Modelling and Simulation in Engineering, volume 2015, pp. 1-15, 2015.

[22] S. Dey, and R. Raikar, "Characteristics of horseshoe vortex in developing scour holes," Journal of Hydraulic Engineering, ASCE, vol. 133, no. 4, pp. 399-413, 2007.

[23] M. Zhao, L. Cheng, and Z. Zang, "Experimental and numerical investigation of local scour around a submerged vertical circular cylinder in steady currents," Coastal Engineering, vol. 57, no. 8, pp. 709-721, 2010. 
[24] D. Liang, L. Cheng, and F. Li, "Numerical modeling of flow and scour below a pipeline in currents. Part II: scour simulation," Coastal Engineering, vol. 52, no. 1, pp. 43-62, 2005.

[25] M. Zhao and L. Cheng, "Numerical modeling of local scour below a piggyback pipeline in currents," Journal of Hydraulic Engineering, ASCE, vol. 134, no. 10, pp. 1452-1463, 2008. 